
\title{
3 Research Square \\ Time to recovery from Proteinuria and its related factors in patients with lupus nephritis
}

\author{
Miramir Aghdashi ( $\nabla$ maaghdashi@umsu.ac.ir) \\ Urmia University of Medical Sciences \\ Khadije Makhdomi \\ Urmia University of Medical Sciences School of Medicine \\ Arash Rashidi \\ Urmia University of Medical Sciences
}

\section{Research article}

Keywords: lupus nephritis, proteinuria, recovery time, predictive factors, Urmia

Posted Date: March 24th, 2020

DOl: https://doi.org/10.21203/rs.3.rs-18751/v1

License: (1) This work is licensed under a Creative Commons Attribution 4.0 International License.

Read Full License

Version of Record: A version of this preprint was published at ACTA MEDICA IRANICA on October 20th, 2021. See the published version at https://doi.org/10.18502/acta.v59i9.7555. 


\section{Abstract}

Background lupus nephritis $(L N)$ is a severe form of systemic lupus erythematosus (SLE) with renal involvement. It affects the kidneys in about $50 \%$ of SLE patients. The aim of this study was to assess the evaluation of proteinuria recovery time and its related factors associated with lupus nephritis patients in Urmia- Northwest of Iran.

Methods A retrospective cohort study was carried out, in which medical records of 80 patients with systemic lupus nephritis referred to Imam Khomeini university hospital were reviewed. According to these records biopsy-proven renal disease has been progressed from September 2009 to September 2013. Proteinuria less than $0.5 \mathrm{~g} / 24 \mathrm{~h}$ was defined as proteinuria recovery. The time elapsed from the diagnosis of proteinuria to its recovery is considered as the duration of proteinuria recovery (month). The findings were analyzed by STATA11 statistical software.

Results Mean age at diagnosis of lupus nephritis was $26.50 \pm 8.10$ years (14-51 years). Mean creatinine level at the start of treatment was $1.20 \pm 0.61 \mathrm{mg} / \mathrm{dl}(0.5-2.80)$. Proteinuria recovery time was 4 months for $25 \%$ of patients, 6 months for $50 \%$ of patients (median time) and 12 months for $75 \%$ of them. Higher class of $L N$ had a trend toward $31 \%$ lower risk of proteinuria recovery (HR: $0.73,95 \% \mathrm{Cl} 0.56-0.96 ; \mathrm{P}=$ $0.02)$, the expected risk is 1.94 times greater in women as compared with men (HR: 1.94, 95\% $\mathrm{Cl} 1.1-3.48$; $\mathrm{P}=0.02)$.

Conclusions The patients in this study population respond to treatment in less time and in comparison with other studies, their proteinuria recovers earlier. Class of lupus nephritis (negative) and gender (positive) were predictive factors proteinuria recovery among LN patients.

\section{Background:}

Systemic lupus erythematosus (SLE) is a chronic inflammatory and autoimmune disease, commonly known as lupus. This disease causes body's immune system to attack its own cells and tissues. According to four COPCORD studies, SLE were detected in $0.06 \%$ of Iranian population (1). Lupus nephritis (LN), with the sign of renal involvement, is one of the most common and serious manifestation of SLE (2). It affects the kidneys in about $50 \%$ of SLE patients (3). Among Iranian population, LN occurred at a young age and mostly in females (6).

Among LN patients, being free from renal flares was associated with attaining Complete Remission (CR) at 12 months after induction therapy (4). According to Hopkins lupus cohort study, renal remission status at 24 months following LN diagnosis is a significant predictor of long-term renal survival (5).

Although potent anti-inflammatory and immunosuppressive therapies end in CKD or ESRD in many patients (3) LN is considered as a poor prognosis indicator and an important risk factor for morbidity and mortality in SLE (3). Approximately, $30 \%$ of patients will progress to end-stage renal disease (ESRD) despite immunosuppressive therapy (6). 
The level of proteinuria at baseline visit predicted the time for improvement. (7) The ability of Proteinuria to change faster at 12 months makes it a favorable endpoint for clinical trials and research studies (8). Proteinuria does not have a significant effect on short-term outcomes (complete or partial or not remission) among Iranian population (9). According to Hopkins lupus cohort data, proteinuria alone was not predictive of ESRD or mortality but was associated with long-term outcomes. Proteinuria mostly predicts long-term renal outcome in lupus nephritis (10).

The main aim of the study was to assess the Evaluation of proteinuria recovery time and its related factors in lupus nephritis patients in Urmia Northwest of Iran.

\section{Methods:}

This retrospective cohort study evaluating of proteinuria recovery time and its related factors associated with lupus nephritis patients in Urmia- Northwest of Iran.

after approval of ethics committee Urmia University of Medical Sciences (No: IR: UMSU.res.1393.207) and obtaining written informed consent, a total number of 80 patients were studied. Medical records of patients with systemic lupus erythematosus were reviewed. According to these records biopsy-proven renal disease has been progressed from September 2009 to September 2013. To be included in the study, patients were required to be diagnosis of lupus nephritis. exclusion criteria were any other disease which accompany with proteinuria similar diabetes. Their process of medical treatment was registered using data recorded in terms of proteinuria.

Proteinuria less than $0.5 \mathrm{~g} / 24 \mathrm{~h}$ was defined as proteinuria recovery. The time elapsed from the diagnosis of proteinuria to its recovery is considered as the duration of proteinuria recovery (month). Demographic and Para clinic characteristics, such as age, gender, base creatinine level, gap between diagnosis of lupus nephritis, chronicity index and class of lupus nephritis were extracted.

Chronicity status was determined based on chronicity index: Glomerular chronicity indices composed of Glomerular sclerosis $0-3$, Fibrous crescents $0-3$, Tubulointerstitial chronicity Interstitial fibrosis $0-3$, Tubular atrophy $0-3$.

The World Health organization (WHO) has divided lupus nephritis into five stages based on biopsy. Classification of lupus nephritis was determined based on the World Health Organization (WHO) recommendations, as pathologic findings. This schema included normal glomeruli (class I), pure mesangial disease (class II), focal proliferative glomerulonephritis (class III), diffuse proliferative glomerulonephritis (class IV), and membranous glomerulonephritis (class V) (2).

Mean \pm SD (Standard deviation) was calculated for continuous variables, and frequencies were measured for categorical variables. Proteinuria recovery time of patients was the primary of the study. The cumulative risk of proteinuria recovery time between two groups was studied by the Kaplan-Meier method. The survival curves of two groups were formally compared by use of the log-rank test. In 
continues predictors (age, based creatinine and Protein level, IN-chronicity status), we considered the Cox proportional hazard model with a single continuous predictor. Predictors with $p$-values less than cut-off value of 0.25 (Univariate Cox regression) were considered to be included in final cox proportional hazards regression analysis to assess the effect of studied factors on protein recovery time in patients. Schoenfeld test was used to assess fitted Cox regression model adequately describes the data. All of analyses were performed by STATA 11 software.

\section{Results:}

This study encompasses 80 patients with lupus nephritis. Mean age at diagnosis of lupus nephritis was $26.50 \pm 8.10$ years (14-51 years). Mean creatinine level at the start of treatment was $1.20 \pm 0.61 \mathrm{mg} / \mathrm{dl}(0.5$ 2.80). Mean Protein level was $2559.31 \pm 1787.65 \mathrm{~g} / 24 \mathrm{~h}$ (600-9500). Lupus nephritis Class I was seen in $1 \%$ of patients, Class II in $28 \%$, Class III in $3 \%$, Class IV in $50 \%$, Class V in $16 \%$. Diffuse proliferative lupus nephritis (Class IV) was the most common type of lupus nephritis among our patients.

The percentile of survival proteinuria recovery time derives from a Kaplan-Meier survivor function Accordingly Proteinuria recovery time was 4 months for $25 \%$ of patients, 6 months for $50 \%$ of patients (median time) and 12 months for $75 \%$ of them (considering the 25 th -50 - 75 th percentiles seen regarding the recovery time) (Table 1 ).

Figure 1 shows duration of proteinuria recovery time for the effect of sex on proteinuria recovery time (Kaplan-Meier method) in patients with lupus nephritis. Median (Q1-Q3) duration of proteinuria recovery time was statistically higher in men than women $(12 ; 4.19-16.48$ vs. $6 ; 4.7-81.19$, log-rank test P-value $=$ $0.04)$.

Table 2 shows Cox regression analysis for duration of protein recovery time using univariate and multivariate analyses. In univariate analyses (unadjusted model), creatinine at initiating treatment, high chronicity scores and class of $L N$, increasing age were associated with $36 \%, 12 \%, 28 \%$ and $1 \%$ lower hazard of proteinuria recovery, respectively.

Final Cox proportional hazards regression analysis higher class of LN had a trend toward $31 \%$ lower hazard of proteinuria recovery, the expected hazard is 1.94 times higher in women as compared to men.

\section{Discussion:}

There are many factors to consider in the time to recovery from proteinuria in patients with lupus nephritis. Recovery proteinuria is one of these factors. Determining the recovery time and investigating the factors affecting it can help patients' treatment process and follow up the response to the treatment.

In this research most of LN patients were females $(1,8,10)$ and male sex was a risk factor of the late proteinuria recovery (7). Grade IV had the highest frequency among patients in this study which is similar to other Iranian studied $(9,11)$. Patients in this study had a lower age average compared to other studies. 
This study is in the same line with other studies in which aging did not have a significant decline in proteinuria recovery $(23,24)$.

In comparison to other studies, the patients responded to treatment in less time and proteinuria recovery happened earlier in this study $(3,6,12)$. Similar to our research, recovery from proteinuria was defined as proteinuria $<0.5 \mathrm{~g} / 24 \mathrm{~h}$ in the study of Touma Z in 2014 (7). Proteinuria recovery time was $52 \%$ within 2 years that is much later than our research and the level of proteinuria at baseline visit predicted the time of improvement. The difference which exits between proteinuria recovery time in this study and other studies may be caused by different methods of patient inclusion such as biopsy, clinical status and laboratorial criteria as in active urine sedimentation simultaneous with proteinuria (6). All the patients in our study reached $<0.5 \mathrm{~g}$ proteinuria in 4 years of follow-up. Touma et al showed that $39 \%$ (84 of 212) of patients in his study still did not get proteinuria recovery in the last visit (after $2.3 \pm 3.2$ years of followup). The reason for this discrepancy is that the recovery time was differently accomplished between the two studies in other hand; Touma et al registered proteinuria recovery only if happened twice respectively whereas in our study one-time proteinuria $<0.5 \mathrm{~g}$ in 24 hours was little enough to categorize patients as recovered from proteinuria. Also in this research the only criteria for protein loss in urine was a 24-hour urine analysis measuring proteinuria but Touma et al have used dip stick or spot ratio of urine creatinine if they did not have access to 24-hour urine samples (6). Hernandez et al (22) showed more similar results with study in which $69 \%$ of patients after 6 months and $86 \%$ after 12 months of treatment beginning were recovered from proteinuria. In another study by Stephen et al, $44 \%$, nearly half of patients, had achieved recovery from proteinuria through $16 \pm 14$ months. The reason for the difference may be the fact that all classes of lupus nephritis were included in our study but others had just studied patients with class IV and V of lupus nephritis. Furthermore, we defined proteinuria $<0.5 \mathrm{~g} / 24$ hours as recovery but in this study proteinuria $\leq 0.33 \mathrm{~g} / 24$ hours was considered as recovery criterion.

Although in this study creatinine of serum and proteinuria at initiation of treatment did not have statistically significant effect on proteinuria recovery, similarly in various studies higher levels of serum creatinine at beginning of treatment were associated with decreased incidence of recovery which is not statistically important (13). Ichinose et al. reports protective effect of lower level of serum creatinine on complete remission at 12 months' follow-up in which duration after renal biopsy was 51 months, which is similar to the definitions of renal remission and follow-up in this study (4). Another research in Iran reported that creatinine level, low GFR and hemoglobin, low C3 and albumin and pathologic Class IV had a significant association with non-remission status among LN patients (9). K Ichinose et al. reported that $\mathrm{CR}$ (complete remission) attaining at 12 months had significantly lower levels of serum $\mathrm{Cr}$ (4). The Hopkins Lupus Cohort (> 500 patients) data results showed that serum creatinine level was in stable range between years 1 to 3 after starting the follow-up for those in CR or PR during 24 months (5). PintoPeñaranda LF in Colombian patients with severe proliferative lupus nephritis reports a baseline creatinine elevation and 24-h proteinuria greater than 1500 mg were statistically significant predictive factors of poor response at 12 months (12). 
According to William a Fung's study serum $\mathrm{Cr}$ and eGFR are fairly stable until 6 years after LN onset, $24 \mathrm{H}$ $\mathrm{P}$ may be more appropriate as a biomarker due to its sensitivity to short-term change than Proteinuria in lupus nephritis (LN) patients (8). Similarly, in another study among Iranian population in Yazd Proteinuria does not have a significant effect on short-term outcomes (complete or partial or not remission) (9).

In this research, high class of $L N$ had a trend toward $31 \%$ lower hazard of proteinuria recovery but this trend was not statistically significant. K Ichinose et al. concluded that classes III and IV of lupus nephritis had less recovery time in the first 12 months of follow-up which is not significant (4). Other studies as in Touma et al. have shown a significant decline in proteinuria recovery incidence as the class of lupus nephritis gets higher (7). Decreasing effect of proteinuria recovery after an increase in the class of lupus nephritis may be due to more renal damage happening in higher classes. Membranous LN (ISN/RPS V) is seen up to $15 \%$ of biopsied SLE patients (14), heavy proteinuria appears in many of Membranous LN and thus a longer period would be necessary to achieve renal remission (3).

In this research, higher Chronicity index was clinically accompanying with lower hazard of proteinuria recovery, but it was not significant. Also similar results were reported in other studies $(4,12)$.

\section{Conclusion:}

we retrospectively analyzed the complete remission rates at 12 months after induction therapy and evaluated the predictive factors for CR and their association with renal flares in patients with LN. We found that patients in our study responded to medical treatment earlier than other studies and proteinuria recovery was achieved in less time. Class of lupus nephritis and gender had significant effect on incidence of proteinuria recovery.

The main strength of this study was strict monitoring of patients. The weaknesses of this study did not evaluate partial remission of patients and Glomerular filtration rate (GFR) as one of complete remission criteria in patients diagnosed with Lupus Nephritis.

\section{Recommendations:}

According to long period of lupus nephritis treatment and the accuracy needed for titration of immunosuppressive drugs and steroids, results of current study can be used for better assessment of drug response in these patients. It is recommended to evaluate the effects of different drugs associated with proteinuria recovery in prospective studies.

\section{Abbreviations}

LN: lupus nephritis, SLE: systemic lupus erythematosus, CR: Complete Remission, ESRD: end-stage renal disease, WHO: The World Health organization,

\section{Declarations}




\section{Ethics approval and consent to participate:}

This study was approved by the Ethics Committee of Urmia University of Medical Science (ID code No: IR.UMSU.1393.207). Informed consent was received for all studied population to their inclusion in the study. details that may reveal the identity of the subjects have been omitted.

\section{Consent for publication:}

all authors It was given according to ethical standards and authorship policy and consent to publish the manuscript in Advances in Rheumatology.

\section{Availability of data and materials:}

The used datasets of this study are available from the corresponding author upon reasonable request.

\section{Funding:}

Funding was provided with research deputy of Urmia university of medical Sciences.

\section{Authors' contributions:}

All of the authors participated in the conception and design of the research, and preparation of the study article.

\section{Acknowledgements:}

This article is results of A.R thesis MD degree. This study was supported by the Urmia research deputy of Urmia University medical sciences. The authors would like to thank Clinical Research Development Unit of Imam Khomeini Hospital for English editing and statistical analyses.

\section{References}

1. Davatchi F, Sandoughi M, Moghimi N, Jamshidi AR, Tehrani Banihashemi A, Zakeri Z, et al. Epidemiology of rheumatic diseases in Iran from analysis of four COPCORD studies. Int $\mathrm{J}$ Rheum Dis. 2016;19(11):1056-62.

2. Nezhad ST, Sepaskhah R. Correlation of clinical and pathological findings in patients with lupus nephritis: a five-year experience in Iran. Saudi J Kidney Dis Transpl. 2008;19(1):32.

3. Almaani S, Meara A, Rovin BH. Update on lupus nephritis. Clin J Am Soc Nephrol. 2017;12(5):825-35.

4. Ichinose K, Kitamura M, Sato S, Eguchi M, Okamoto M, Endo Y, et al. Complete renal response at 12 months after induction therapy is associated with renal relapse-free rate in lupus nephritis: a singlecenter, retrospective cohort study. Lupus. 2019;28(4):501-9.

5. Davidson JE, Fu Q, Ji B, Rao S, Roth D, Magder LS, et al. Renal Remission Status and Longterm Renal Survival in Patients with Lupus Nephritis: A Retrospective Cohort Analysis. J Rheumatol. 
2018;45(5):671-7.

6. Obrișcă B, Jurubiță R, Andronesi A, Sorohan B, Achim C, Bobeica R, et al. Histological predictors of renal outcome in lupus nephritis: the importance of tubulointerstitial lesions and scoring of glomerular lesions. Lupus. 2018;27(9):1455-63.

7. Touma Z, Urowitz MB, Ibañez D, Gladman DD. Time to Recovery from Proteinuria in Patients with Lupus Nephritis Receiving Standard Treatment. J Rheumatol. 2014;41(4):688.

8. Fung WA, Su J, Touma Z. Predictors of Good Long-Term Renal Outcomes in Lupus Nephritis: Results from a Single Lupus Cohort. Biomed Res Int. 2017;2017.

9. Soleymani Salehabadi H, Bashiri H, Nouri Majelan N, Dehghan A, Owlia M. Prognostic factors of lupus nephritis in an Iranian population. Rheumatology Research. 2017;2(1):17-22.

10. Tamirou F, Lauwerys BR, Dall'Era M, Mackay M, Rovin B, Cervera R, et al. A proteinuria cut-off level of $0.7 \mathrm{~g} /$ day after 12 months of treatment best predicts long-term renal outcome in lupus nephritis: data from the MAINTAIN Nephritis Trial. Lupus Science \& Medicine. 2015;2(1):e000123.

11. Akbarian M, Faezi ST, Gharibdoost F, Shahram F, Nadji A, Jamshidi AR, et al. Systemic lupus erythematosus in Iran: a study of 2280 patients over 33 years. Int J Rheum Dis. 2010;13(4):374-9.

12. Pinto-Peñaranda LF, Duque-Caballero V, Márquez-Hernández JD, Muñoz-Grajales C, VelásquezFranco CJ. Predictive factors for low rate of remission in a population of Colombian patients with severe proliferative lupus nephritis. Clin Rheumatol. 2015;34(5):897-903.

13. Korbet SM, Lewis EJ. Complete remission in severe lupus nephritis: assessing the rate of loss in proteinuria. Nephrology Dialysis Transplantation. 2011;27(7):2813-9.

14. Lusco MA, Fogo AB, Najafian B, Alpers CE3. AJKD Atlas of Renal Pathology: Membranous Lupus Nephritis, ISN/RPS Class V. Am J Kidney Dis. 2017;70(2):e13-e5.

\section{Tables}

Table 1: Clinical characteristics of patients with lupus nephritis at the time of renal biopsy 


\begin{tabular}{|c|c|c|}
\hline \multicolumn{2}{|l|}{ Variables } & $\mathrm{N}(80)$ \\
\hline \multicolumn{2}{|l|}{ Males (\%) } & $16(20)$ \\
\hline \multicolumn{2}{|l|}{ Age $($ Mean \pm Sd $)$} & $26.50 \pm 8.10$ \\
\hline \multicolumn{2}{|l|}{ Creatinine Level At Recovery Time (Mg/Dl) } & $1.16 \pm 0.7$ \\
\hline \multicolumn{2}{|l|}{ Based Creatinine Level (Mg/Dl) } & $1.20 \pm 0.61$ \\
\hline \multicolumn{2}{|l|}{ Proteinuria At Initiating Treatment (Mg/24h) } & $2559.31 \pm 1787.65$ \\
\hline \multicolumn{2}{|c|}{ Gap Between Proteinuria And Diagnosis Of Lupus Nephritis(Years) } & $1.92 \pm 3.62$ \\
\hline \multirow[t]{5}{*}{ Class Of Lupus Nephritis(\%)* } & I & $1(1)$ \\
\hline & $\mathrm{Ii}$ & $22(28 \%)$ \\
\hline & Iii & $2(3)$ \\
\hline & Iv & $40(50)$ \\
\hline & $\mathrm{V}$ & $13(16)$ \\
\hline \multirow[t]{8}{*}{ Ln-Chronicity Status $\infty$} & 0 & $8(10.4)$ \\
\hline & 1 & 11(14.3) \\
\hline & 2 & $28(36.4)$ \\
\hline & 3 & $14(18.2)$ \\
\hline & 4 & $10(13)$ \\
\hline & 5 & $1(1.3)$ \\
\hline & 6 & $4(5.2)$ \\
\hline & 9 & $1(1.3)$ \\
\hline
\end{tabular}

*2 patients had mixed class, $\infty$ Chronicity Index

Table 2: Cox regression analysis for duration of protein recovery time using univariate and multivariate model 


\begin{tabular}{|c|l|c|}
\hline \multirow{2}{*}{ Variables } & \multicolumn{1}{|c|}{ Univariate } & Multivariate $\infty$ \\
\cline { 2 - 3 } & Hazard ratios CI* $(95 \%) ; P$ value & Hazard ratios CI (95\%) \\
\hline Creatinine at initiating treatment $(\mathrm{mg} / \mathrm{dl})$ & $0.64(0.44-0.92) ; 0.01$ & $0.81(0.53-1.22) ; 0.31$ \\
\hline LN-chronicity status* & $0.88(0.76-1.02) ; 0.1$ & $0.95(0.81-1.10) ; 0.48$ \\
\hline Proteinuria at initiating treatment $(\mathrm{mg} / 24 \mathrm{~h})$ & $1.00(0.99-1.0002) ; 0.44$ & \\
\hline Age & $0.99(0.96-1.01) ; 0.54$ & $0.73(0.56-0.96) ; 0.02$ \\
\hline Class of lupus nephritis & $0.72(0.57-0.92) ;<0.01$ & $1.94(1.1-3.48) ; 0.02$ \\
\hline Sex (female) & $1.71(0.97-2.98) ; 0.06$ & \\
\hline
\end{tabular}

${ }^{*} \mathrm{CI}=$ confidence Interval,${ }^{* *}$ Chronicity Index,$\infty$ backward selection of significant variables

\section{Figures}

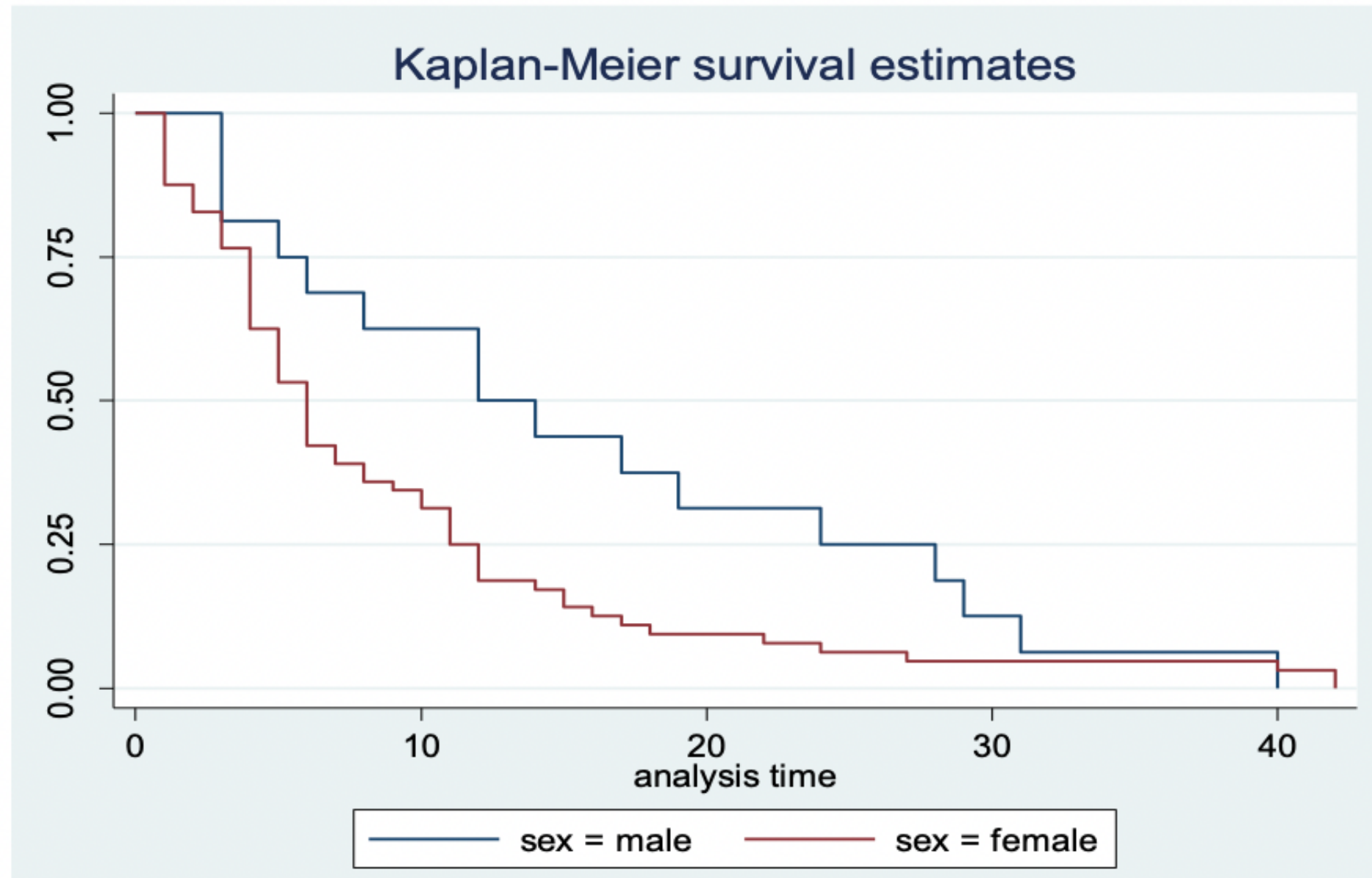

Figure 1 
Kaplan- Meier method for the effect of sex on proteinuria recovery time in patients with lupus nephritis 